\title{
Impact of robot-assisted surgery appearance on reduction of annual blood transfusion cases in Japan: application of meta-analysis and NDB open data
}

\author{
Hirohito Imada ${ }^{1} \cdot$ Tomoyuki Akita $^{1}$ (D) Aya Sugiyama ${ }^{1} \cdot$ Junko Tanaka $^{1}$
}

Received: 9 November 2021 / Accepted: 30 December 2021 / Published online: 8 January 2022

(c) The Author(s), under exclusive licence to Springer-Verlag London Ltd., part of Springer Nature 2022

\begin{abstract}
In Japan, the robot-assisted partial nephrectomy (RAPN) started to be covered by health insurance since 2016, and it is replacing conventional open partial nephrectomy (OPN). RAPN is a minimally invasive surgery, and the spreading of RAPN in partial nephrectomy (PN) performed annually is expected to reduce the number of blood transfusions in Japan. The number of PN surgery in Japan was calculated using the Japanese NDB open data in 2018. We extracted articles comparing the transfusion rates of RAPN and OPN from 2017 to 2021 using PubMed, Web of Science, and Ichu-shi, and integrated the ratios of transfusion rates by meta-analysis. We estimated the reduction in the annual transfusion cases in PN due to the widespread use of RAPN. The total number of renal cancer surgeries in 2018 was 21,298, of which 3,876 (18.2\%) were RAPN and 4,384 (20.6\%) were OPN. For the comparison of transfusion implementation rate between RAPN and OPN, 871 articles were screened and 27 articles were included. The pooled ratio in transfusion rate of RAPN compared with OPN was 0.49 [0.46, 0.52]; the introduction of RAPN was estimated to have reduced the annual number of transfusions in PN by $9.1 \%$ compared with that of unintroduced RAPN. This study showed quantitatively evaluated the impact of the introduction of RAPN on the decrease in the annual number of transfusions in Japan. This method has the potential to evaluate the impact of robot-assisted surgery on the use of blood products for transfusion.
\end{abstract}

Keywords Renal cancer $\cdot$ Robot-assisted partial nephrectomy $\cdot$ Open partial nephrectomy $\cdot$ Transfusion · Transfusion reduction rate

\section{Introduction}

In Japan, 7.52 million operations alongside with 1.13 million blood transfusions are performed annually [1]. In Japan, the demand for blood products for transfusion is expected to increase [2], and since the number of people who can donate blood is decreasing [3], a shortage of blood donations in future has been suggested [4]. Furthermore, the pandemic of a new coronavirus in December 2019 has been suggested to reduce the number of blood donors and affect the supply of blood products for transfusion [5].

Tomoyuki Akita

tomo-akita@hiroshima-u.ac.jp

1 Department of Epidemiology, Infectious Disease Control and Prevention, Graduate School of Biomedical and Health Sciences, Hiroshima University, 1-2-3, Kasumi, Minami-ku, Hiroshima 734-8551, Japan
Recent years, laparoscopy has become the common choice for gastrointestinal surgery, and robot-assisted surgery (RAS), an extension of laparotomy, is becoming more popular. In Japan, RAS was first covered for prostate cancer in 2012, it was covered for renal cancer in 2016.

The most recent meta-analysis comparing OPN and LPN transfusion rates was reported in 2020, and reported a 0.8 lower transfusion rate for OPN compared to LPN [6]. On the other hand, comparison of blood transfusion rates between open surgery (OS) and RAS has been reported previously [7]. The meta-analyses of blood transfusion rates have been conducted [8-11] in recent years, but the most latest study summarized the clinical studies published up to 2016. However, there has been no quantitative study computing and illustrating how much the number of blood transfusions during surgery has decreased due to the replacement of partial nephrectomy by RAS. Therefore, our study aimed to evaluate the reduction the blood transfusions cases due to appearance of RAS quantitatively, by 
application the concept of Population Attributable Fraction (PAF).

In this study, we calculated the difference and ratio of transfusion rates between RAPN and OPN in PN by meta-analysis of additional studies conducted after 2016, and attempted to calculate "the percentage of reduction in transfusion cases that the introduction of insurance coverage for RAPN contributed to the reduction in transfusion rates in $\mathrm{PN}$ ".

\section{Study design and methods}

\section{Difference in transfusion rate between RAPN and OPN}

\section{Literature search method}

We have conducted a systematic database search of research literature which aimed to compare transfusion rate of RAPN and OPN through PubMed, Web of Science, and Ichu-shi (Japanese) and the study timespan covered from January 1, 2017 to March 30, 2021. The main keywords used for literature search were robot-assisted surgery, surgery, and kidney cancer, and the detailed search equation were formulated as "("da Vinci" OR robotic OR computer-assisted) AND (surgery) AND (kidney cancer [Mesh])". As for literatures on meta-analysis published during this period, primary researches in the meta-analysis were included to the systematic review, additionally.

\section{Inclusion/exclusion criteria}

The inclusion criteria cover the studies that compared the surgical outcomes of RAPN and OPN, or also describing the transfusion rate. The review literatures, commentaries, and study protocols were excluded.

The literature was reviewed independently by $\mathrm{HI}$ and TA, and when judgments on the discrepancy selection of papers were done by AS.

\section{Quality assessment}

All studies were evaluated according to "the 2011 level of evidence for therapy studies" as follows: from level 1 (systematic review of randomized trials or n-of-1 trials) to level 5 (mechanism-based reasoning). Quality of reporting on the outcome data was evaluated according to the Martin criteria [12].

\section{Meta-analysis}

Difference and ratio of transfusion rates between RAPN and OPN were calculated by Mantel-Haenszel method. To assess the study heterogeneity, we calculated Cochran's $Q$ test and $I^{2}$ value. Evaluation of publication bias was assessed by Funnel plot and Egger's regression.

\section{Expected reducing proportion of blood transfusions cases in PN surgery by the introduction of RAPN}

\section{Calculation of the number of surgeries for RAPN and OPN}

The targeted cases were identified using the international classification of diseases (ICD-11). We calculated the number of surgeries for PN (K773, K773-2, K773-3, and K773-5) by procedure (OPN, RAPN, and LPN) in Japan using the 5th NDB Open Data (April 1, 2018 to March $31,2019)$.

\section{"The subtracted transfusion fraction" (STF) in PN surgery by the introduction of RAPN}

"The population attributable fraction" $(P A F)$ is the proportion of incident cases or deaths that could be prevented if a risk factor could be eliminated.

In this study, we applied the concept of $P A F$ to calculate "the Subtracted Transfusion Fraction" (STF), which is the expected reducing proportion of transfusion cases since the introduction of RAPN, based on the "Ratio of Transfusion rate" $\left(R R_{R A P N / O P N}\right)$ calculated by Meta-analysis and the proportion of each procedure (OPN $p_{1}, \operatorname{RAPN} p_{2}, \mathrm{LPN} p_{3}$, $\left.p_{1}+p_{2}+p_{3}=1\right)$ calculated from NDB open data. STF was calculated by the following formula (Figure 1).

$\mathrm{STF}_{R A P N / O P N}=\left(1-\mathrm{RR}_{R A P N / O P N}\right) \times p_{2}$

In other words, the $\mathrm{STF}_{R A P N / O P N}$ in this study is the percentage of transfusion rate avoided in RAPN compared with that in OPN.

The transfusion rate between OPN and LPN was assumed 0.8 times as high as the rate, which was reported by You's study [6]. Similarly, $\mathrm{STF}_{L P N / O P N}$ by LPN was calculated as follows:

$\mathrm{STF}_{L P N / O P N}=\left(1-\mathrm{RR}_{L P N / O P N}\right) \times p_{3}$ 
Transfusion cases

Transfusion cases under an assumption all surgeries were OPN

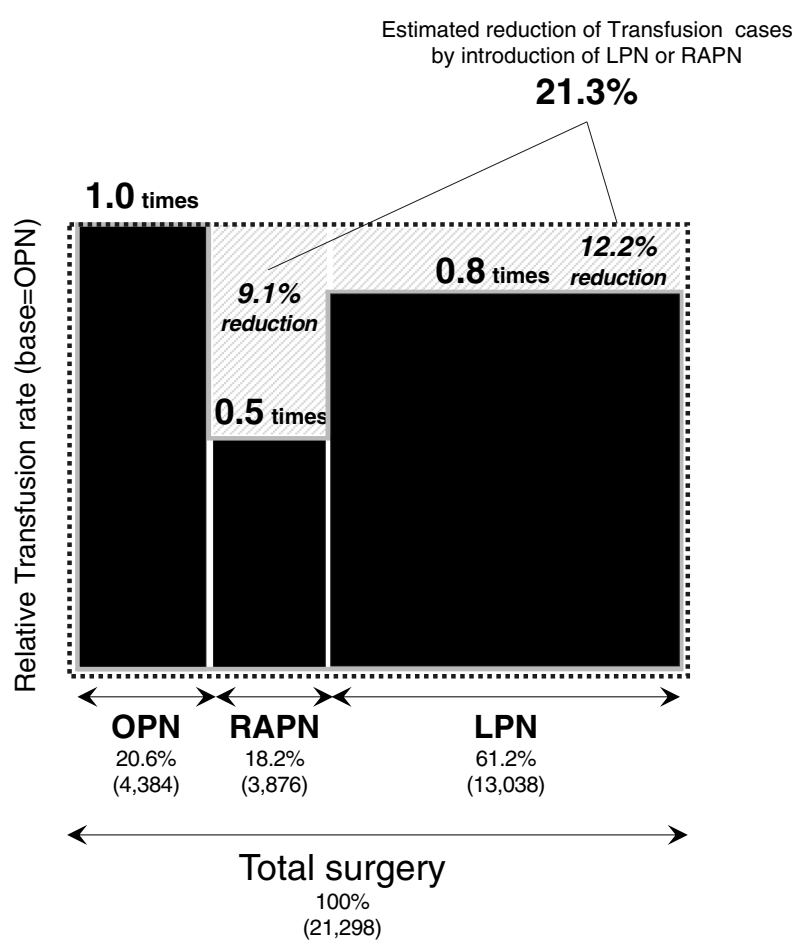

Fig. 1 Reduction of transfusion cases by introduction of laparoscopic partial nephrectomy and robot-assisted partial nephrectomy. The vertical axis is the relative transfusion rate based on OPN. The horizontal axis is the number and percentage of surgeries for OPN, RAPN, and LPN, respectively, in Japan in 2018. The black area is the number of transfusions performed, and the gray area is the number of transfusions that would have been required if all renal cancer removal surgeries had been OPN. In other words, the gray area shows the number of transfusions reduced by the introduction of RAPN and LPN. OPN Open partial nephrectomy, RAPN Robot-assisted partial nephrectomy, $L P N$ Laparoscopic partial nephrectomy

\section{Ethical consideration}

As this study is based on published literature and open data, no ethical issues are applicable.

\section{Results}

\section{Systematic review}

The details of the search chart are shown in Supplementary Figure 1. A total of 871 papers were extracted by the search keywords. In addition, we added 84 references in three Meta-analysis. We finally identified 27 literatures which met the inclusion criteria absolutely. All 27 articles had evidence level 3 , with 4 studies scoring 8 or higher on the
Martin Criteria. All included articles provided the sample of 37,423 RAPNs and 23,750 OPNs (Supplementary Table 1) and were included in this meta-analysis.

\section{Meta-analysis}

The pooled ratio in transfusion rate of RAPN compared with OPN was 0.49 [95\% confidence interval (CI) 0.46, 0.52] (Supplementary Figure 2). The heterogeneities of the studies were high in pooled ratio (Cochran's $Q$ test $p<0.01$, $I^{2}$ value $=72 \%$ ). Funnel plots showed no asymmetry in the evaluation of publication bias (Supplementary Figure 3). Egger's regression analysis showed no significant publication bias ( $p=0.44$ for pooled ratio).

\section{Expected reducing proportion of blood transfusions cases in PN by the introduction of RAPN}

\section{Number of surgeries for RAPN and OPN}

Based on the calculation using the 5th NDB open data, the total number of PN surgeries from April 1, 2018 to March 31,2019 was 21,298, with 3876 (18.2\%) RAPNs, 4384 (20.6\%) OPNs, and 13,038 (61.2\%) LPNs.

\section{"The Subtracted Transfusion Fraction" (STF) in PN surgery by the introduction of RAPN}

It was estimated that the introduction of RAPN and LPN for renal cancer removal reduced the number of blood transfusions by $21.3 \%$ ( $9.1 \%$ for RAPN and $12.2 \%$ for LPN) compared to the assumption of no introduction (OPN in all cases) (Figure 1).

In other words, the number of transfusions performed could have been $1 /(1-0.213)=1.27$ times higher if all renal cancer surgeries in 2018 had been performed by OPN.

\section{Discussion}

For the meta-analysis, three papers from 2016 onward were added, and the transfusion ratios and transfusion rates of RAPN and OPN were confirmed to be the same as the results of the meta-analysis published in 2019[11].

Although RAPN accounted for only $18.2 \%$ of all renal cancer resections in Japan, the introduction of RAS was estimated to have reduced the number of transfusions performed by $9 \%$ of all transfusions, compared with the number of transfusions that would have been performed if all procedures had been performed by OPN.

In Japan, after 6 years of RAS insurance coverage for prostate cancer, $72 \%$ of procedures were performed with RAS [1]. According to the treatment guideline for prostate 
cancer, the ratio of transfusion rate between RAS and OS for prostate cancer removal is 0.2 times, and the reduction rate of transfusion rate is $57.6 \%$, which indicates that it has greatly contributed to the decrease in the number of transfusions performed. RAS is becoming widespread, not only for renal cancer, but also for other cancers, and advances in medical technology have the advantage not only of minimizing invasiveness for patients, but also of reducing the use of blood products.

In addition, the number of blood donors is expected to decrease due to the pandemic of the new coronavirus infection starting in 2019, and this research method and results can be used as basic data to examine the shortage of blood products for transfusion and blood donation promotion measures.

In this study, the number of surgeries was calculated using the NDB open data. For the protection of personal information, aggregations of less than 10 cases were counted as 0 cases. In addition, because the name of the aggregated diagnosis was "receipt description," some of the RAPNs may be described as LPNs in this study. Therefore, the actual number of RAPNs may be higher. If the ratio of the transfusion rate due to the introduction of a new technology can be clarified, the rate of decrease in the annual number of transfusions can be estimated using the same method as in this study. While the decrease in the transfusion rate due to the introduction of RAPN was estimated by meta-analysis in this study, but more detailed verification may be possible by conducting analysis using individual receipt databases such as NDB.

Supplementary Information The online version contains supplementary material available at https://doi.org/10.1007/s11701-021-01365-7.

Acknowledgements This work was supported by MHLW Research on Regulatory Science of Pharmaceuticals and Medical Devices Program Grant Number JPMH21KC1005. We thank KoKo of Hiroshima University, Hiroshima, Japan, for advice on English proofreading.

Author contributions Concept JT, HI, TA; literature search HI, TA; data extraction HI, TA, AS; data analysis HI, TA; critical review HI, TA, AS, JT.

\section{Declarations}

Conflict of interest All authors certify that they have no affiliations with or involvement in any organization or entity with any financial interest or non-financial interest in the subject matter or materials discussed in this manuscript.

\section{References}

1. Ministry of Health Labour and Welfare (2020) The 5th NDB Open Data. https://www.mhlw.go.jp/stf/seisakunitsuite/bunya/00001 77221_00008.html. Accessed 01 Oct 2021

2. Akita $T$ et al (2016) Predicting future blood supply and demand in Japan with a Markov model: application to the sex- and agespecific probability of blood donation. Transfusion 56(11):2750 2759. https://doi.org/10.1111/trf.13780

3. Ministry of Health Labour and Welfare (2021) Blood Donation Promotion 2025. https://www.mhlw.go.jp/stf/newpage_19547. html. Accessed 11 July 2021

4. Japan Red Cross (2010) Simulation of the number of blood products supplied for transfusion and blood donors based on the estimated future population in Japan. https://www.mhlw.go.jp/stf2/ shingi2/2r9852000000styz-att/2r9852000000su6y.pdf. Accessed 01 Oct 2021

5. Shan H, Zhang P (2004) Viral attacks on the blood supply: the impact of severe acute respiratory syndrome in Beijing. Transfusion 44(4):467-469. https://doi.org/10.1111/j.0041-1132.2004. 04401.x

6. You C et al (2020) Laparoscopic versus open partial nephrectomy: a systemic review and meta-analysis of surgical, oncological, and functional outcomes. Front Oncol 10:583979. https://doi.org/10. 3389/fonc. 2020.583979

7. Novara G et al (2012) Systematic review and meta-analysis of perioperative outcomes and complications after robot-assisted radical prostatectomy. Eur Urol 62(3):431-452. https://doi.org/ 10.1016/j.eururo.2012.05.044

8. Shen $\mathrm{Z}$ et al (2016) The comparison of perioperative outcomes of robot-assisted and open partial nephrectomy: a systematic review and meta-analysis. World J Surg Oncol 14(1):220. https://doi.org/ 10.1186/s12957-016-0971-9

9. Xia L, Wang X, Xu T, Guzzo TJ (2017) Systematic review and meta-analysis of comparative studies reporting perioperative outcomes of robot-assisted partial nephrectomy versus open partial nephrectomy. J Endourol 31(9):893-909. https://doi.org/10.1089/ end.2016.0351

10. Grivas N et al (2019) Robot-assisted versus open partial nephrectomy: comparison of outcomes. A systematic review. Minerva Urol Nefrol 71(2):113-120. https://doi.org/10.23736/S0393-2249. 19.03391-5

11. Tsai SH et al (2019) Open versus robotic partial nephrectomy: Systematic review and meta-analysis of contemporary studies. Int J Med Robot 15(1):e1963. https://doi.org/10.1002/rcs.1963

12. Martin RC 2nd, Brennan MF, Jaques DP (2002) Quality of complication reporting in the surgical literature. Ann Surg 235(6):803813. https://doi.org/10.1097/00000658-200206000-00007

Publisher's Note Springer Nature remains neutral with regard to jurisdictional claims in published maps and institutional affiliations. 